\title{
Delayed Infrarenal Aortic Pseudoaneurysm Treated by Endovascular Stent Graft in Pyogenic Spondylitis
}

\author{
Jae-Hyuk Shin ${ }^{1}$, Dae-Hyun Hwang ${ }^{2}$, Chae-Hyun Pang ${ }^{1}$, Shaobai Wang ${ }^{3}$, \\ In-Sung Kim ${ }^{1}$, Jung-Tae Ahn ${ }^{1}$, Young-Woo Kim ${ }^{1}$, Ho-Guen Chang ${ }^{1}$ \\ ${ }^{1}$ Department of Orthopaedic Surgery, Musculoskeletal Center, Hallym University Dongtan Sacred Heart Hospital, \\ Hallym University College of Medicine, Hwaseong, Korea \\ ${ }^{2}$ Department of Radiology, Hallym University Dongtan Sacred Heart Hospital Korea, Hallym University College of Medicine, Hwaseong, Korea \\ ${ }^{3}$ Bioengineering Laboratory, Department of Orthopaedic Surgery, Massachusetts General Hospital, Harvard Medical School, Boston, MA, USA
}

A 61-year-old male patient with pyogenic spondylodiscitis and epidural and psoas abscesses underwent posterior decompression, debridement, and instrumented fusion, followed by anterior debridement and reconstruction. Sudden onset flank pain was diagnosed 7 weeks postoperatively and was determined to be a pseudoaneurysm located at the aorta inferior to the renal artery and superior to the aortic bifurcation area. An endovascular stent graft was applied to successfully treat the pseudoaneurysm. Postoperative recovery was uneventful and infection status was stabilized.

Keywords: False aneurysm; Endovascular procedure; Stents; Spondylitis

\section{Introduction}

Anterior debridement and reconstruction remains the mainstay of surgical treatment for pyogenic spondylodiscitis [1]. Vascular injury to a large vessel is a lethal complication following an anterior spinal surgery approach. Large vessel injuries during spine operations have been reported during posterior discectomy, anterior discectomy, and corpectomy [2,3]. Vascular complications may include vessel lacerations or formation of a pseudoaneurysm or arteriovenous fistula. These complications potentially threaten life due to hemodynamic instability or create a large scar after open surgical treatment. A pseudoaneurysm is a defect in the vascular wall leading to an extravascular pulsating hematoma or is covered by a fibrous wall, whereas a true aneurysm has an intact attenuated arterial wall. Endovascular stent grafts have been successfully used to treat vascular injuries [4]. However, the management and indication for stent grafting remains controversial when underlying infection is present $[5,6]$. No report of endovascular stent grafting during pyogenic spondylitis is available. Here, we describe the delayed appearance of an infra-renal aortic pseudoaneurysm following anterior surgery for pyogenic spondylitis, which was treated successfully with an endovascular stent graft.

\section{Case Report}

A 61-year-old male patient presented with severe back

Received Jun 16, 2012; Revised Jul 28, 2012; Accepted Aug 13, 2012

Corresponding author: Ho-Guen Chang

Department of Orthopaedic Surgery, Musculoskeletal Center, Hallym University Dongtan Sacred Heart Hospital, Hallym University College of Medicine, 40 Samsung 1-ro 3-gil, Hwaseong 445-170, Korea

Tel: +82-31-8086-2412, Fax: +82-31-8086-2438, E-mail: hgchang@hallym.or.kr 
pain for 15 days, which occurred even during rest. A physical examination showed tenderness in the back are, and difficulty changing positions due to pain. Motor and sensory functions were normal. Erythrocyte sedimentation rate (ESR) and C-reactive protein (CRP) were elevated to $88 \mathrm{~mm} / \mathrm{hr}$ and $88.2 \mathrm{mg} / \mathrm{L}$ (normal, $<8.0 \mathrm{mg} / \mathrm{L}$ ) respectively. Lumbar spine magnetic resonance imaging
(L-MRI) findings showed disk space narrowing and an endplate irregularity with a hyperintense signal on T2weighted images at the L1-2 and L3-4 disk levels, as well as diffuse swelling in prevertebral and paraspinal soft tissue at the lumbar spine level, which suggested early findings of infective spondylodiscitis (Fig. 1A). The patient underwent conservative treatment with teicoplanin
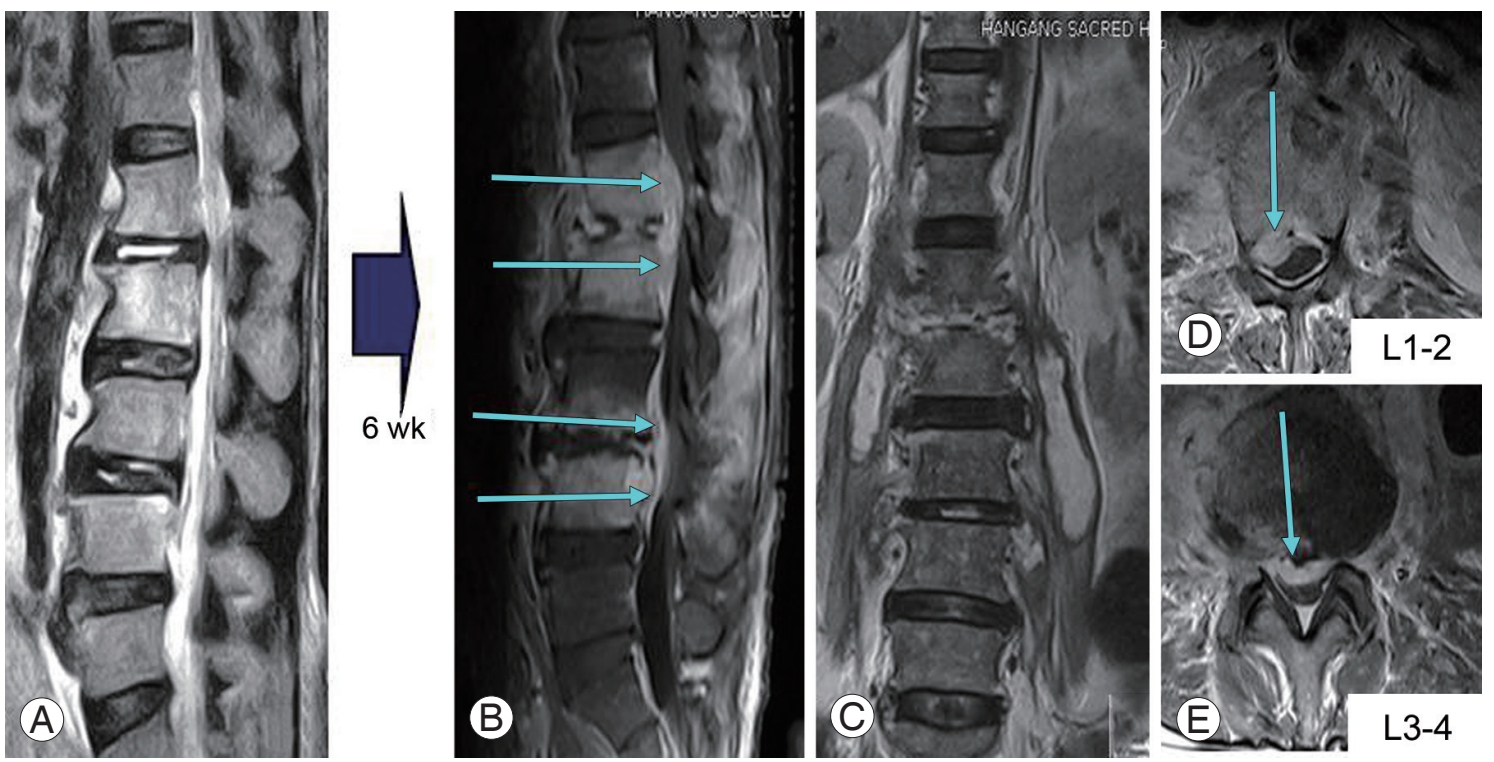

Fig. 1. Initial magnetic resonance imaging. Under the impression of infective spondylitis (A), conservative treatment with teicoplanin was applied. Six weeks later, the infection progressed to form spondylitis, epidural abscess, and intra-psoas abscess (B-E).
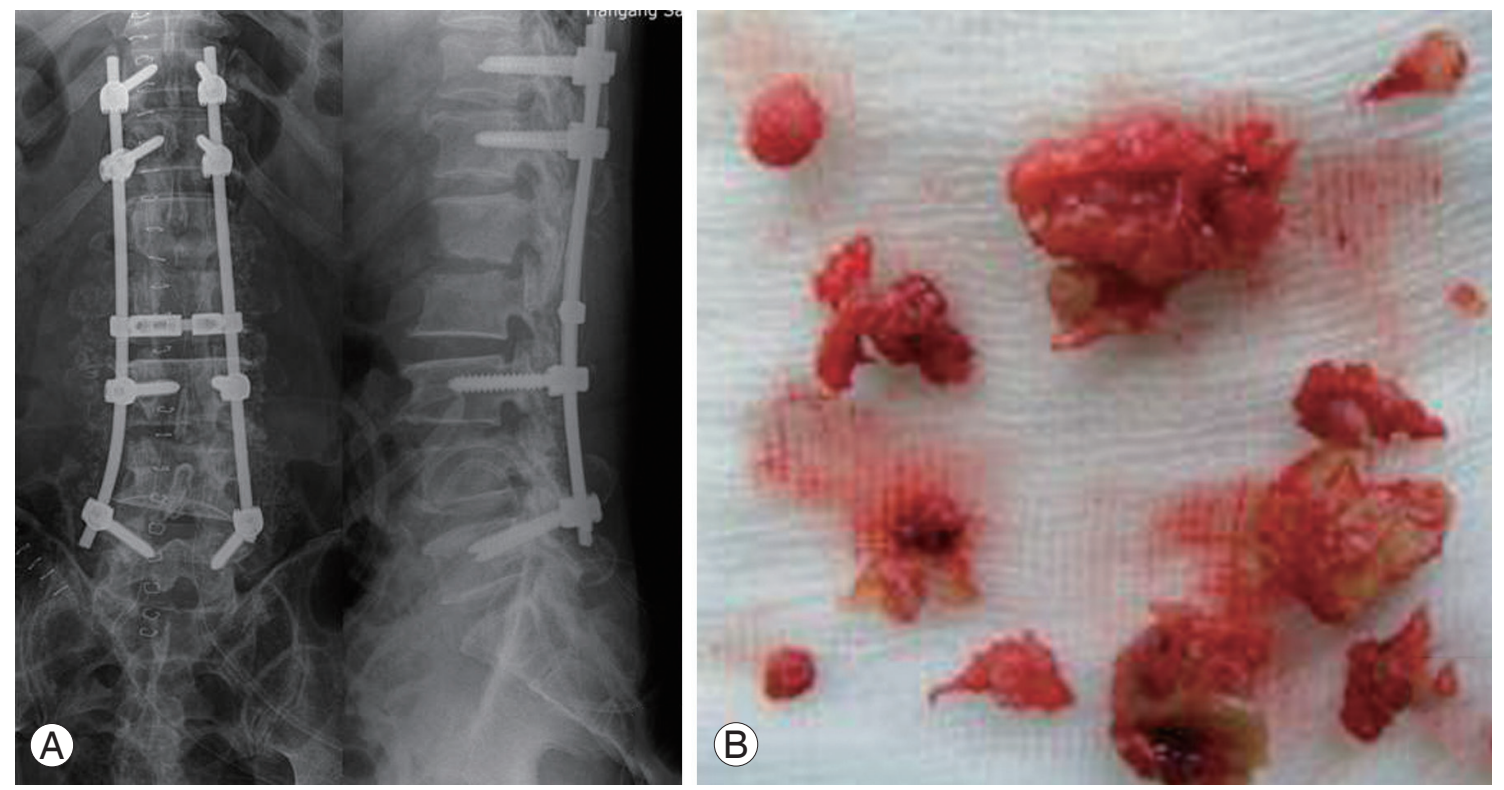

Fig. 2. Decompressive laminectomy and irrigation drainage were done from $L 1$ through $L 3$. Posterior instrumented fusion was done from T11 through L5 with autogenous iliac bone graft (AIBG). L3-4 was approached through facetectomy, extensively debrided, and reconstructed with strut AIBG (A, B). We posteriorly drained the psoas abscess by approaching between the transverse processes, and by vertically splitting the psoas muscle in meticulous manner to protect the lumbar nerve roots. 
antibiotic for 6 weeks.

However symptoms did not improve, and motor power of both low extremities was weakened to grade IV with persistent radiating pain. Laboratory results showed an increased ESR to $130 \mathrm{~mm} / \mathrm{hr}$ and CRP of $111 \mathrm{mg} / \mathrm{L}$. A blood culture was positive for methicillin-resistant Staphylococcus aureus (MRSA), and a follow-up L-MRI demonstrated progression of the pyogenic spondylitis (Fig. 1B-E). The patient underwent a staged operation. Posterior decompressive laminectomy at the abscess level (L13 ), drainage of the epidural abscess, and posterior instru- mented fusion (T11-L5) were performed initially (Fig. 2 ). The psoas abscess was partially drained posteriorly by approaching posteriorly through the intertransverse membrane, followed by longitudinal splitting of the psoas muscle, which was targeted on MRI images. The intraoperative specimen culture result was positive for MRSA. Debridement of the infected tissue and reconstruction with an autogenous iliac strut bone graft was performed 2 weeks after the initial surgery using an anterior retroperitoneal approach (Fig. 3). ESR and CRP were $81 \mathrm{~mm} /$ $\mathrm{hr}$ and $9.43 \mathrm{mg} / \mathrm{dL}$ at 4 weeks postoperatively. The patient
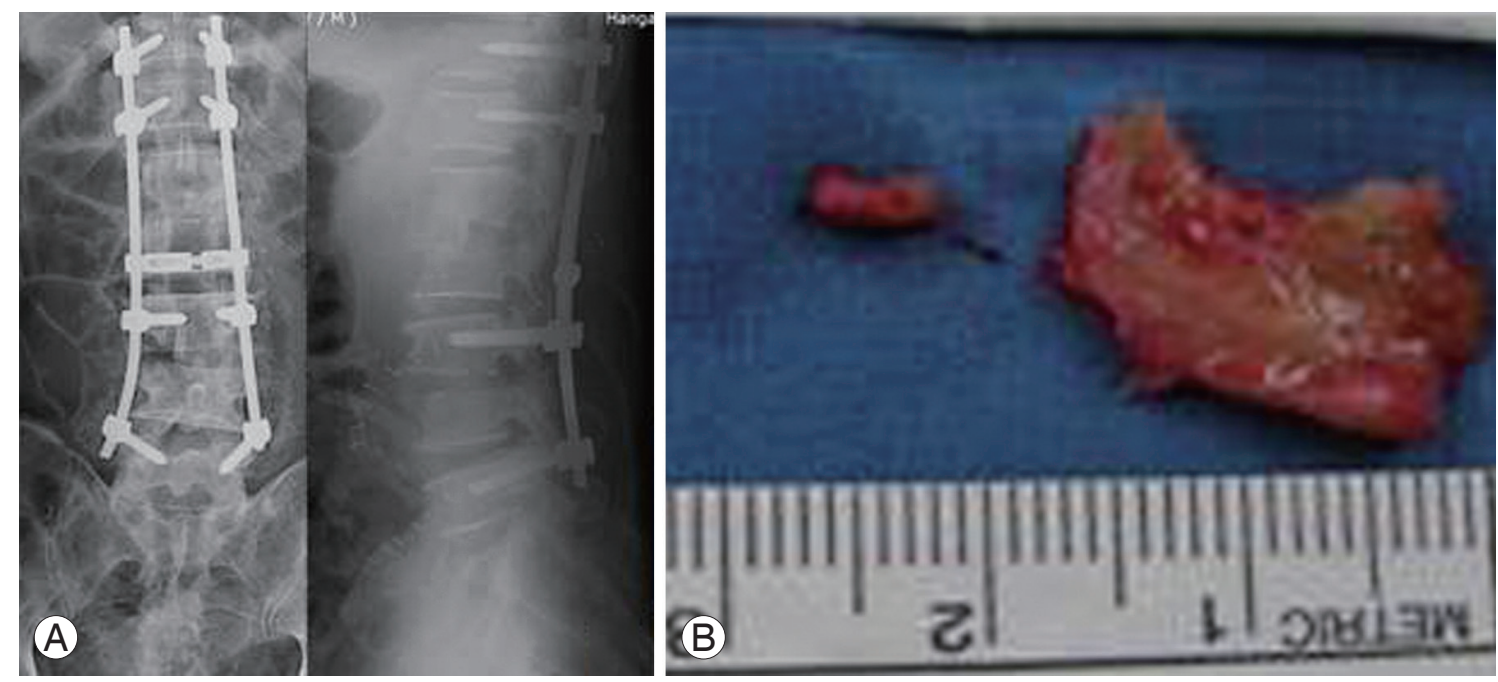

Fig. 3. Two weeks after the initial operation, L1-2, and L2-3 discs were anteriorly approached, debrided, and reconstructed with strut autogenous iliac bone graft (A, B).

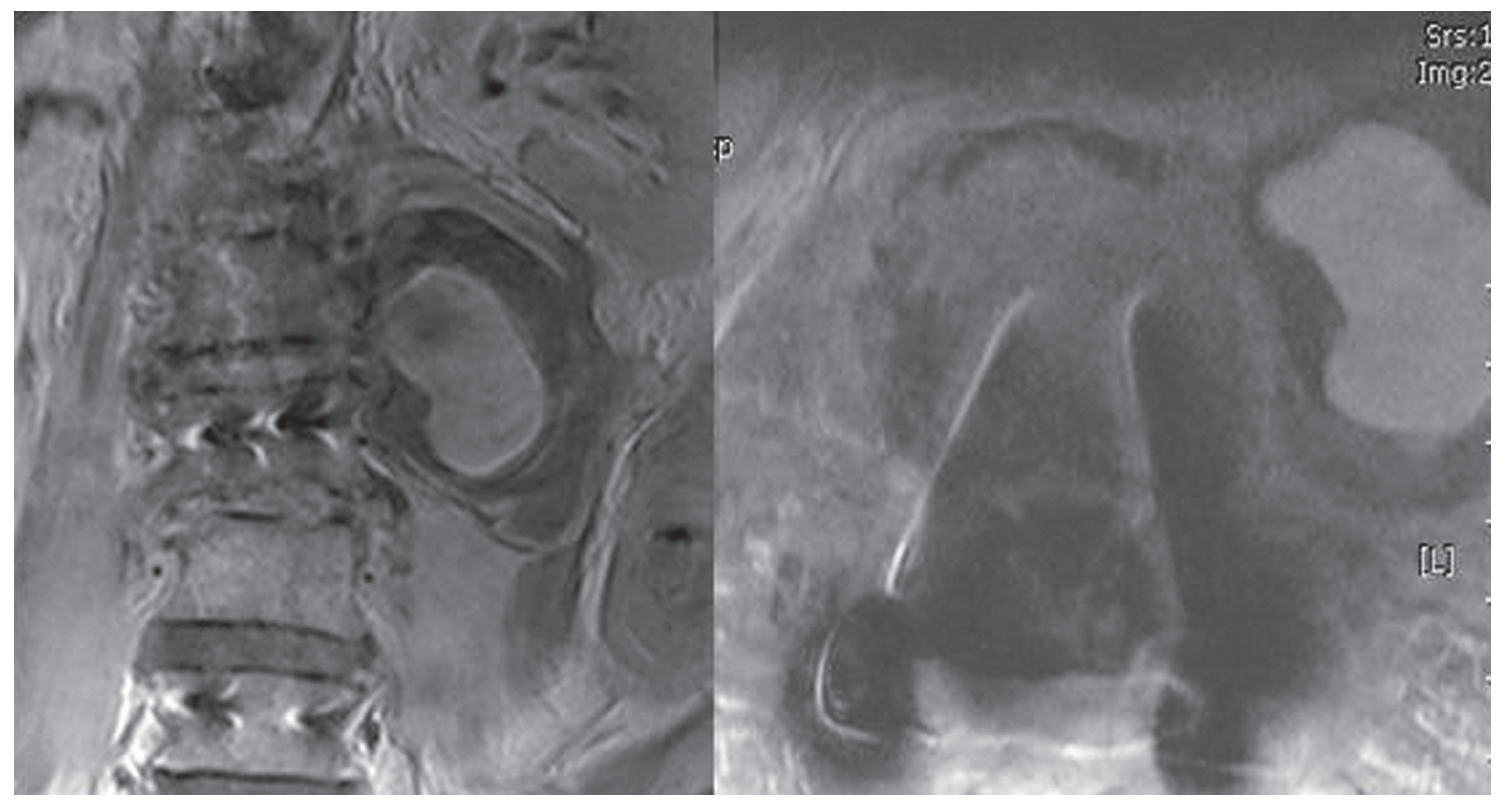

Fig. 4. At postoperative 7 weeks, lumbar spine magnetic resonance imaging with contrast image showed hematoma like lesion which was later diagnosed as a pseudoaneurysm. 
developed relative sudden discomfort on the left flank area over a 3 day period at 7 weeks postoperatively. ESR and CRP were $71 \mathrm{~mm} / \mathrm{hr}$, and $37.9 \mathrm{mg} / \mathrm{dL}$, respectively. A contrast L-MRI showed the lesion as a hematoma-like dilatation (Fig. 4). Diagnostic angiography demonstrated a weakly enhanced, pulsating dilatation without definite arterial wall involvement, which was $6.4 \mathrm{~cm} \times 4.5 \mathrm{~cm}$ in size. We radiographically diagnosed the lesion as a pseudoaneurysm located in the infrarenal aortic area (Fig. 5). An endovascular stent graft (SEAL stent graft type I; 2.4 $m m \times 6 \mathrm{~cm}$; S \& G Biotech Inc., Seoul, Korea) was applied. The celiac trunk, the superior mesenteric artery, and both renal arteries were preserved, whereas the inferior mesenteric artery was blocked (Fig. 6). The flank pain disappeared immediately after applying the graft. However, Klebsiella pneumoniae was detected in blood and urine cultures, so we administered intravenous meropenem according to the sensitivity test, which controlled the infection. The antibiotic was continued for 4 weeks until the CRP value normalized. Thereafter, infection control was

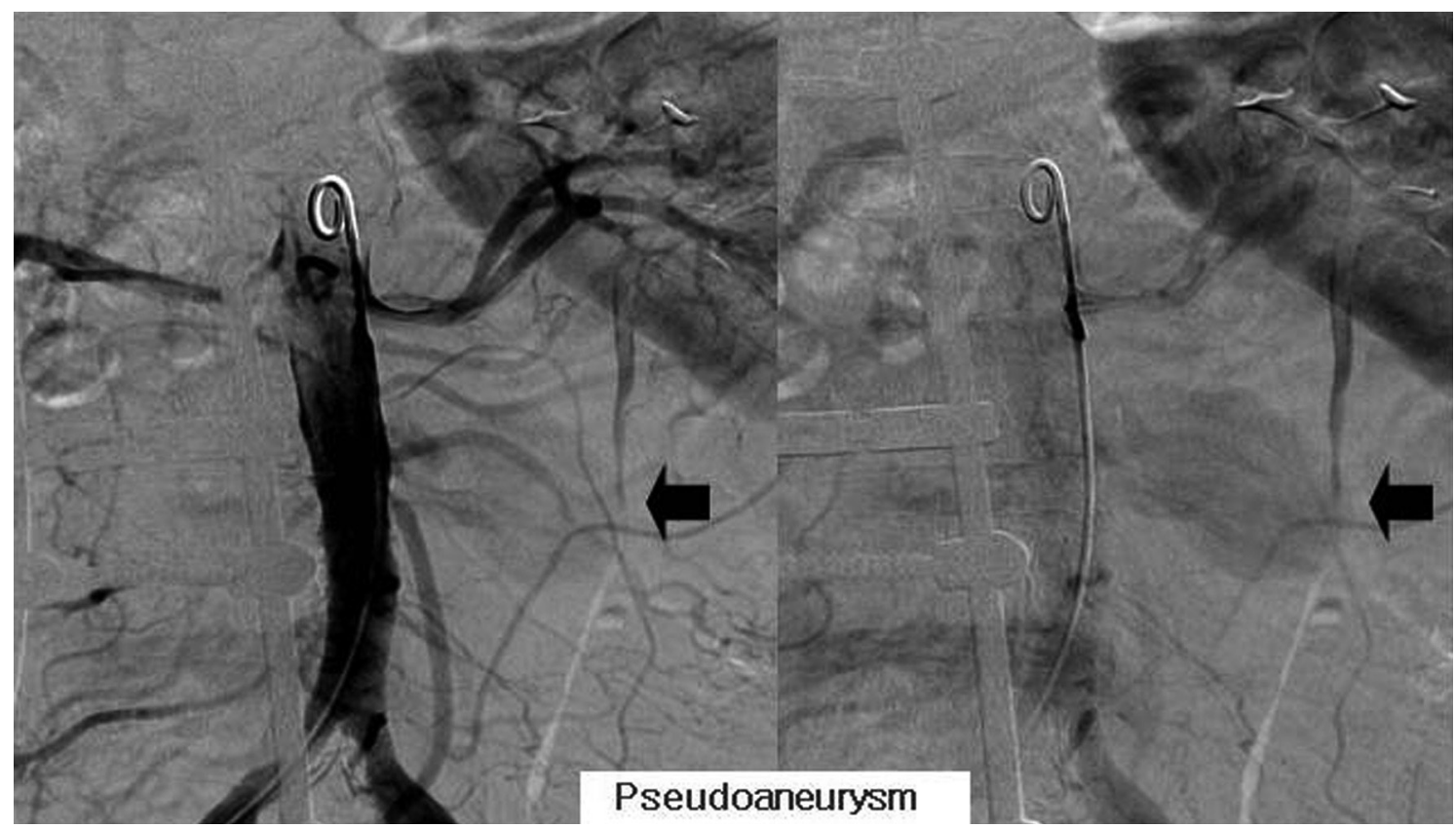

Fig. 5. Diagnostic angiogram demonstrated $6.4 \times 4.5 \mathrm{~cm}$ sized pseudoaneurysm originating from the left lower infrarenal abdominal aortic area (arrow).
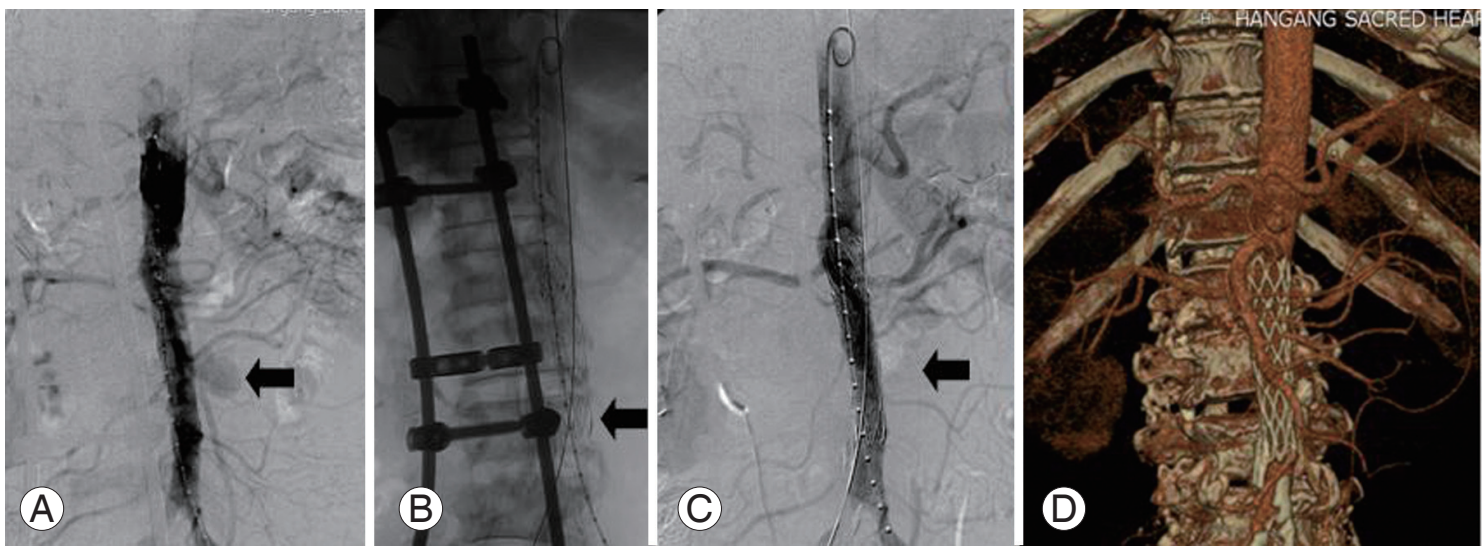

Fig. 6. Endovascular stent graft using SEAL Stent (S\&G Biotech Inc.) was applied (2.4 mm×6 cm). The psudoaneurysm (A) radiographically disappeared immediately after inserting the stent graft $(\mathbf{B}, \mathbf{C})$, obviating the symptom as well. Computed tomography angiogram follow-up (D) of the stent graft confirmed the adequate position. The celiac trunk, the superior mesenteric artery, and the renal arteries were preserved. 
uneventful. Laboratory findings remained stable at the 6 month follow-up (ESR, $64 \mathrm{~mm} / \mathrm{hr}$; CRP, $3.70 \mathrm{mg} / \mathrm{dL}$ ), and at the 3 and one-half year follow-up (ESR, $35 \mathrm{~mm} /$ $\mathrm{hr}$; CRP, $2.08 \mathrm{mg} / \mathrm{dL}$ ). The patient has maintained normal daily life without medication (Fig. 7).

\section{Discussion}

We report a unique clinical case of a delayed aortic pseudoaneurysm combined with pyogenic spondylitis that was successfully treated using an endovascular stent graft.

Pyogenic spondylodiscitis of the spine is a serious illness and remains a difficult treatment challenge. The presence of neurological deficit, epidural and psoas abscesses, intractable pain, and aggravation with medical treatment made surgical treatment necessary in this case. The mainstay for treating pyogenic spondylodiscitis is anterior debridement and reconstruction with a strut graft combined with posterior instrumented fusion [1].

Vascular injury is a potential but major and lethal complication following anterior or posterior spinal procedures. Perioperative vascular injuries are classified at the time of recognition: intraoperative or immediate postoperative, which represents postoperative 45 minutes to 24 hours; and delayed, which represents postoperative several days to 8 years [7]. Delayed vascular injury, usually a pseudoaneurysm, may develop rather slowly with signs of audible murmur, edema, and cool lower extremities. Characteristics of a pseudoaneurysm may also

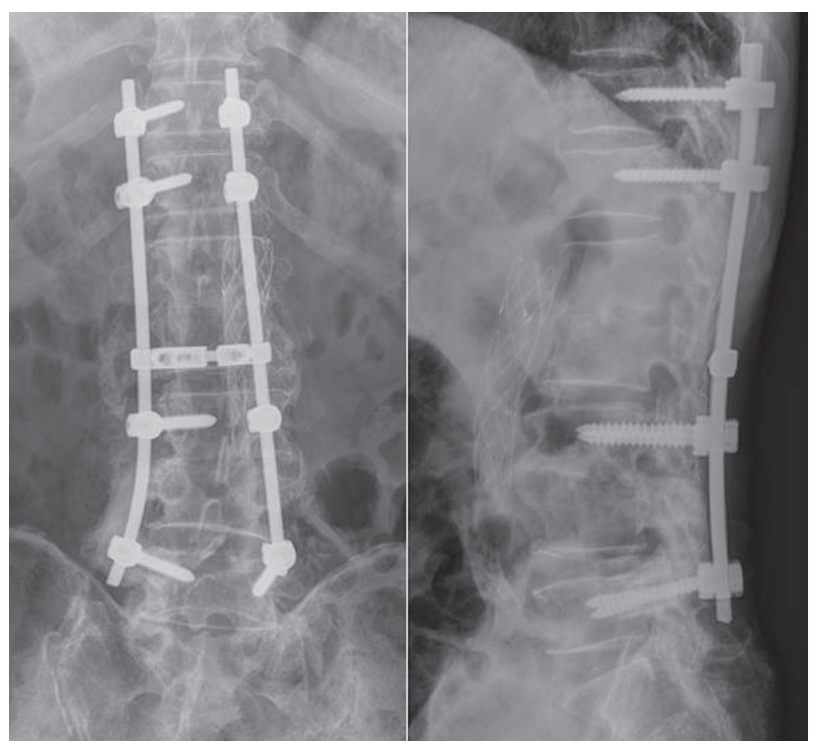

Fig. 7. At postoperative 3 years 6 months follow-up, the instruments were maintained well and the patient was back to normal daily life. include rapid enlargement of a pulsating vascular malformation, and locating the lesion is important before the procedure. An arterio-venous fistula following a discectomy of the common iliac artery and common iliac vein is successfully treated using an endovascular stent graft [8]. An intraoperative approach to a ruptured common iliac artery can be attempted through the popliteal artery under ultrasound guidance with the pateint in the prone position [7]. A descending thoracic aortic aneurysm of $10 \mathrm{~cm}$ in length, which was treated with an endovascular stent graft of $40 \mathrm{~mm}$ in diameter and $12 \mathrm{~cm}$ in length, caused ischemia at the T6-T12 level, and paraplegia [9]. Preservation of the artery of Adamkiewicz is important to maintain blood circulation to the spinal cord during stenting in the thoracic region [10].

Although inserting an endovascular stent graft during an infection is controversial, a MRSA-infected pseudoaneurysm at the proximal iliac artery after bilateral total hip and knee replacement in juvenile-onset rheumatoid arthritis has been treated successfully both for pseudoaneurysm and for infection. Chronic exposure to MRSA infection was suggested as the cause of a pseudoaneurysm in a patient with chronic osteomyelitis of the femur [5].

In the present case, although intraoperative bleeding control was stable, persistent exposure to a MRSA infection from a psoas abscess in combination with an infected vertebral or disc space is thought to have eroded the vessel wall leading to the development of the delayed pseudoaneurysm. The patient suddenly complained of left flank pain at 7 weeks postoperatively. The initial differential diagnosis included recurrence of the abscess. Contrast-enhanced MRI and angiography confirmed the diagnosis of aortic pseudoaneurysm. The lesion was located inferior to the renal arteries, and the stent was safely located without compromising the major blood supply of the celiac trunk or blocking the superior mesenteric and renal arteries. Prolonged exposure to MRSA could erode a weak portion of the vessel wall to cause a pseudoaneurysm in a pateint with pyogenic spondylodiscitis and a psoas abscess [5]. Thus, a high index of suspicion is necessary to detect a delayed aortic pseudoaneurysm. K. pneumoniae in the blood and urine was considered an opportunistic infection, as the clinical symptoms and laboratory findings improved without remarkable additional clinical findings.

Further study is required for safe application of a SEAL stent graft in infected tissue. Endovascular stent grafting 
may be region dependent to avoid blocking major aortic branches or too many segmental arteries, including the Adamkiewicz artery, around the spinal cord. Intraoperative accessibility may well be rewarded for prompt and efficient application of these procedures.

In summary, we present successful treatment of a delayed infrarenal pseudoaneurysm during treatment of pyogenic spondylodiscitis, which was successfully treated with an endovascular stent graft. We emphasize that a high index of suspicion is required for detecting a lesion with a delay period, and that locating the lesion in the vessel is an important factor to use an endovascular stent graft.

\section{Conflict of Interest}

No potential conflict of interest relevant to this article was reported.

\section{References}

1. Dimar JR, Carreon LY, Glassman SD, Campbell MJ, Hartman MJ, Johnson JR. Treatment of pyogenic vertebral osteomyelitis with anterior debridement and fusion followed by delayed posterior spinal fusion. Spine (Phila Pa 1976) 2004;29:326-32.

2. Zahradnik V, Kashyap VS. Alternative management of iliac vein injury during anterior lumbar spine exposure. Ann Vasc Surg 2012;26:277.e15-8.
3. Wood KB, Devine J, Fischer D, Dettori JR, Janssen M. Vascular injury in elective anterior lumbosacral surgery. Spine (Phila Pa 1976) 2010;35:S66-75.

4. Patel SN, Kettner NW. Abdominal aortic aneurysm presenting as back pain to a chiropractic clinic: a case report. J Manipulative Physiol Ther 2006;29:409.e1-7.

5. Clarke MG, Thomas HG, Chester JF. MRSA-infected external iliac artery pseudoaneurysm treated with endovascular stenting. Cardiovasc Intervent Radiol 2005;28:364-6.

6. Sanada J, Matsui O, Arakawa F, et al. Endovascular stent-grafting for infected iliac artery pseudoaneurysms. Cardiovasc Intervent Radiol 2005;28:83-6.

7. Karaikovic EE, Rattner Z, Bilimoria MM, et al. Coil embolization of a lumbar artery to control vascular injury during intradiscal surgery. Spine (Phila $\mathrm{Pa}$ 1976) 2010;35:E163-6.

8. Momoh AO, Barshes NR, Kougias P, Huynh TT, Lin $\mathrm{PH}$. Endovascular treatment of postlaminectomy aortic pseudoaneurysm. J Vasc Surg 2008;47:1083-5.

9. Lee MY, Lee MG, Hong KS, et al. A case of paraplegia following endovascular stent repair of descending thoracic aortic aneurysm. Korean Circ J 2011;41:1603.

10. Uotani K, Yamada N, Kono AK, et al. Preoperative visualization of the artery of Adamkiewicz by intraarterial CT angiography. AJNR Am J Neuroradiol 2008;29:314-8. 